\title{
Bond formation dynamics of dicyanoaurate in the first excited singlet state
}

\author{
So Hyeong Sohn and Taiha Joo* \\ Department of Chemistry, Pohang University of Science and Technology (POSTECH), Pohang \\ 37673, South Korea
}

\begin{abstract}
Time-resolved fluorescence of $\left[\mathrm{Au}(\mathrm{CN})_{2}{ }^{-}\right]_{3}$ in water reveals a coherent vibration of $74 \mathrm{~cm}^{-1}$. A massive non-Condon effect, proved unambiguously by time-resolved fluorescence spectra, demonstrates that the wave packet motion arises from the coherent bond formation.
\end{abstract}

\section{Introduction}

Bond making and breaking is one of the fundamental processes in chemical reactions. A viable method to study bond formation is to exploit a gold complex using its nature, aurophilicity. It was reported that $\mathrm{Au}(\mathrm{CN})_{2}{ }^{-}$, one of the simplest gold complexes, exists as a trimer in aqueous solution at $0.3 \mathrm{M}$. It was reported that the trimer, $\left[\mathrm{Au}(\mathrm{CN})_{2}^{-}\right]_{3}$, has a loose bent structure in the ground state, whereas it has a tight staggered structure in the triplet state by forming covalent bonds [1].

Recently, there have been controversies on the process of bond formation and the excitation wavelength for the trimer. Iwamura et al. claimed that bond shortening occurs during the intersystem crossing to the triplet state within $0.5 \mathrm{ps}$ followed by bent-to-linear transformation. They employed transient absorption (TA) spectroscopy with the pump wavelength of $310 \mathrm{~nm}$ to excite the trimer exclusively [2, 3]. However, Kim et al. suggested in their time-resolved X-ray solution scattering (TRXSS) study that the bent-to-linear transformation and bond contraction occur simultaneously within $0.5 \mathrm{ps}$, and further bond shortening takes place within $1.6 \mathrm{ps}[4,5]$. They also confirmed the same results for different excitation wavelengths at both 266 and $310 \mathrm{~nm}$.

The signals of TA and TRXSS include information from undesired states such as ground state, non-photoexcited state, and other higher excited states, and they are intermingled with that from the intended $\mathrm{S}_{1}$ state. In this regards, time-resolved fluorescence (TF) may play an important role to reveal the bond formation dynamics of the $\mathrm{S}_{1}$ state and to resolve the controversy because the TF signals come from the first excited singlet state exclusively. Furthermore, accurate time constants and coherent nuclear wave packet oscillations can be attained by TF with high enough time resolution.

\section{Results and discussion}

\footnotetext{
*Corresponding author: thjoo@postech.ac.kr
} 

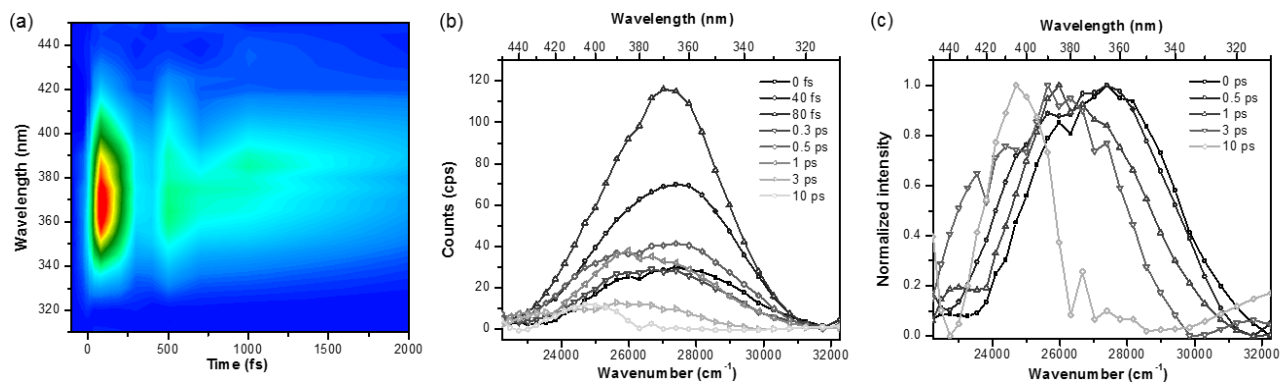

Fig. 1. Time-resolved fluorescence spectra $\mathrm{Au}(\mathrm{CN}){ }_{2}{ }^{-} 0.3 \mathrm{M}$ aqueous solution represented by (a) contour plot, (b) raw data, and (c) normalized intensity plot for better comparison.

Time-resolved fluorescence spectra (TFS) of $\mathrm{Au}(\mathrm{CN})_{2}{ }^{-}$aqueous solution $(0.3 \mathrm{M})$ is shown in Fig. 1. The overall intensity of the TFS oscillates at early times, which indicates that the usual Condon approximation is no longer valid. This shows that origin of the oscillation of the TF intensity is not modulation of the transition energy but modulation of the oscillator strength of the emitting state. The non-Condon effect can also be verified by the fact that the phases of the oscillations in the TF signals measured at the high and low frequency sides of the fluorescence band center are the same. Fig. 1b-c show that TFS is centered around $370 \mathrm{~nm}$ at early times $(<1 \mathrm{ps}$ ), and the emission band at $400 \mathrm{~nm}$ survives for a long time ( $>10 \mathrm{ps}$ ). The $370 \mathrm{~nm}$ band is broad, whereas the $400 \mathrm{~nm}$ band is relatively narrow, which implies that there may be more than one conformers at early times but only one rigid conformer survives at later times. TFS were fitted globally using a minimal set of time constants to give lifetimes of $1.5 \mathrm{ps}$ and $\sim 1 \mathrm{~ns}$ for the 370 and $400 \mathrm{~nm}$ bands, respectively. It was reported that both the 370 and $400 \mathrm{~nm}$ bands come from the triplet state $[1,2]$. The 370 band, however, shows considerable signal intensity in spontaneous time-resolved fluorescence, that is, oscillator strengths are sizable, and we can conclude that this band arise from the $S_{1}$ state. The $400 \mathrm{~nm}$ band comes from $T_{1}$ state, because of the strong spinorbit coupling of gold atom.
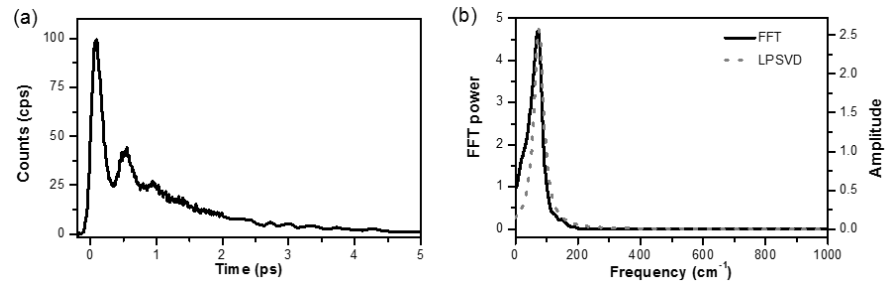

Fig. 2. (a) Time-resolved fluorescence at a fixed wavelength $(350 \mathrm{~nm})$. (b) Frequency spectra obtained by fast Fourier Transform (solid line) and linear prediction singular value decomposition (LPSVD) (dashed line).

$\mathrm{TF}$ at a fixed wavelength was obtained with the instrument response function (IRF) of 85 fs (FWHM) to measure the vibrational frequencies as shown in Fig. 2. The oscillation decays extremely fast with the dephasing time of $\sim 250 \mathrm{fs}$. The TF signal fits well to a sum of an exponential of $1.5 \mathrm{ps}$ time constant and a damped sinusoid of $74 \mathrm{~cm}^{-1}$. The $1.5 \mathrm{ps}$ lifetime is three times longer than the value reported previously $(0.52 \mathrm{ps})$ [2]. In fact, a time constant of 0.5 ps was obtained when we intentionally lower the time resolution to $280 \mathrm{fs}$ and fitted the TF signal to a single exponential. As shown in Fig. 2b, frequency analyses using fast Fourier transform (FFT) and linear prediction singular value decomposition (LPSVD) methods also gave one vibrational mode at $74 \mathrm{~cm}^{-1}$. Considering three gold atoms, there are three vibrational modes that can be assigned to the $74 \mathrm{~cm}^{-1}$ mode. 
According to the quantum chemical calculations (TDDFT/ $\omega$ B97XD with basis set of AVTZ-PP for gold atoms, and AVDZ for $\mathrm{C}$ and $\mathrm{N}$ atoms), frequencies of the bending, symmetric stretching, and asymmetric stretching modes are $\sim 14,102$, and $152 \mathrm{~cm}^{-1}$, respectively. Asymmetric stretching could be eliminated from the candidates because its frequency is too high compared with the experimental value. Although there are large displacements between the ground state and the first excited state in terms of the bending and stretching motions, that is, those vibrational modes should be excited strongly in $\mathrm{S}_{1}$ state, we expect that the period of the bending motion is too long to be detected in $\mathrm{TF}$. Thus, the $74 \mathrm{~cm}^{-1}$ can be assigned to the symmetric stretching vibration in the $\mathrm{S}_{1}$ state.

We have performed several control experiments to confirm the origin of the fluorescence of gold trimer for the excitation wavelengths of 266 and $310 \mathrm{~nm}$. Firstly, we obtained the full range of emission spectrum to examine the presence of the dimer emission. It was reported that the emission band of the dimer is centered at $280 \mathrm{~nm}$, whereas the isomers of trimer contribute to the peaks at 325 and $393 \mathrm{~nm}$ [1]. According to the TFS, emission bands are observed at 370 and $400 \mathrm{~nm}$ only, which should be assigned to the isomers of the trimer. Secondly, the concentration dependence of TF was performed. At a low concentration $(0.04 \mathrm{M})$, where the gold complex exists as a dimer predominantly, TF signals are exactly the same as those obtained at $0.3 \mathrm{M}$ including the nuclear wave packet oscillations, although TF intensity decreased drastically. Finally, excitation wavelength dependence of TF was also carried out. We obtained TF and TFS at two different excitation wavelengths of 270 and $305 \mathrm{~nm}$. Again, TF gave the same oscillation frequency and lifetimes, and TFS shows the $370 \mathrm{~nm}$ band, which proves that the emission that we observe must be originated from first excited singlet state of $\left[\mathrm{Au}(\mathrm{CN})_{2}^{-}\right]_{3}$ in aqueous solution.

\section{Conclusion}

We have observed the dynamics of the $\mathrm{S}_{1}$ state of $0.3 \mathrm{M}\left[\mathrm{Au}(\mathrm{CN})_{2}{ }^{-}\right]_{3}$ aqueous solution with high time resolution TF experiment. TFS show that the emission originates from two conformers in the excited state. A large amplitude nuclear wave packet motion was observed in the $\mathrm{S}_{1}$ state, which was assigned to the $\mathrm{Au}-\mathrm{Au}$ symmetric stretching vibration. The vibrational mode displays massive non-Condon effect. During the bond formation (large displacement), transition dipole moment should change significantly to give the large non-Condon effect. We resolved most of the controversies in the literature by the unique feature of $\mathrm{TF}$ experiments, and revealed detailed bond formation dynamics of the gold trimer.

\section{References}

1. M.A. Rawashdeh-Omary, M.A. Omary, H.H. Patterson, J.P. Fackler, J. Am. Chem. Soc. 123, 11237 (2001).

2. M. Iwamura, K. Nozaki, S. Takeuchi, T. Tahara, J. Am. Chem. Soc. 135, 538 (2013)

3. M. Iwamura, R. Wakabayashi, J. Maeba, K. Nozaki, S. Takeuchi, T. Tahara, Phys. Chem. Chem. Phys. 18, 5103 (2016)

4. K.H. Kim, J.G. Kim, S. Nozawa, T. Sato, K.Y. Oang, T.W. Kim, H. Ki, J. Jo, S. Park, C. Song, T. Sato, K. Ogawa, T. Togashi, K. Tono, M. Yabashi, T. Ishikawa, J. Kim, R. Ryoo, J. Kim, H. Ihee, S.-i. Adachi, Nature 518, 385 (2015)

5. K.H. Kim, J.G. Kim, K.Y. Oang, T.W. Kim, H. Ki, J. Jo, J. Kim, T. Sato, S. Nozawa, S.-i. Adachi, H. Ihee, Struct. Dyn. 3, 043209 (2016) 